\title{
Use of bisphosphonates and the risk of osteonecrosis among cancer patients: a systemic review and meta-analysis of the observational studies
}

\author{
Si-Huei Lee • Rai-Chi Chan • Shy-Shin Chang • \\ Yin-Ling Tan • Kai-Hsiang Chang • Matthew C. Lee • \\ Huai-En Chang $\cdot$ Chien-Chang Lee
}

Received: 4 January 2013 / Accepted: 8 October 2013 /Published online: 8 November 2013

(C) Springer-Verlag Berlin Heidelberg 2013

\begin{abstract}
Purpose This study aims to systematically review observational studies evaluating the use of bisphosphonates (BPs) and risk of osteonecrosis of the jaw (ONJ) among cancer patients.

Methods PubMed, Embase, and Cochrane Library were screened from database inception to Aug 2012. Two reviewers independently identified cohort and case-control studies evaluating the use of oral or intravenous (IV) BPs and the risk of ONJ and extracted the characteristics of the studies and risk estimates. Pooled estimates of odds ratios and $95 \%$ confidence intervals were derived by random effects metaanalysis. Subgroup analyses were carried out according to patients' characteristics and route of BP use.
\end{abstract}

Electronic supplementary material The online version of this article (doi:10.1007/s00520-013-2017-y) contains supplementary material, which is available to authorized users.

S.-H. Lee • R.-C. Chan $\cdot$ K.-H. Chang

Department of Rehabilitation and Physical Medicine, Taipei Veteran

General Hospital, Taipei, Taiwan

S.-H. Lee • R.-C. Chan

Department of Rehabilitation and Physical Medicine, National

Yang-Ming University, Taipei, Taiwan

\section{S.-S. Chang}

Department of Family Medicine, Chang Gung Memorial Hospital,

Taoyuan, Taiwan

\section{S.-S. Chang}

College of Medicine, Chang Gung University, Taoyuan, Taiwan

\section{S.-S. Chang}

Graduate Institute of Clinical Medical Sciences, College of

Medicine, Chang Gung University, Taoyuan, Taiwan
Results We identified eight studies, including 1,389 cases and 569,620 controls. Use of BPs was associated with a significantly increased risk of ONJ (odds ratio (OR) 4.25; $95 \%$ confidence interval (CI) $3.67-5.36 ; I^{2}=0 \%$ ). The summary OR was 4.22 (95\% CI 3.21-5.54; $I^{2}=0 \%$ ) for adjusted studies. IV BPs were associated with higher risk (OR 4.27; $95 \%$ CI 3.38-5.40; $I^{2}=0 \%$ ) than oral BPs (OR 1.18; $95 \%$ CI $0.89-1.56$; $I^{2}=0 \%$ ). Hospital-based studies were associated with higher risk estimates than population-based studies.

Conclusion The available evidence suggests that use of BPs in cancer patients is associated with a substantial risk for ONJ. Patients receiving IV BP are at the highest risk. It is important to assess oral health before initiating therapy and to avoid dental procedures during the active phase of intravenous BP therapy.

Y.-L. Tan

Department of Medicine, College of Medicine, National Taiwan University Hospital, Taipei, Taiwan

M. C. Lee

Medical Wisdom Consultants, Houston, USA

C.-C. Lee $(\square)$

Department of Emergency Medicine, National Taiwan University Hospital Yunlin Branch, No. 579, Sec. 2, Yunlin Road, Douliou,

Yunlin County 640, Taiwan

e-mail: medicalpaper@gmail.com

C.-C. Lee

Department of Epidemiology, Harvard School of Public Health, Boston, USA

H.-E. Chang

Department of Medicine, National Yang-Ming University, Taipei, Taiwan 
Keywords Bisphosphonate · Osteonecrosis · Cancer · Meta-analysis

\section{Introduction}

Bisphosphonates (BPs) are synthetic analogs of a naturally occurring pyrophosphate that makes bone less susceptible to bone resorption by reducing the survival of osteoclasts and modulating the signaling from osteoblasts to osteoclasts [1]. Clinically, BPs are indicated for the management of metastatic cancer in bone, prevention of osteoporosis and related fracture, treatment of Paget's disease of bone, and acute management of hypercalcemia. Since 2003, case reports and case series of osteonecrosis of jaw (ONJ) have emerged, and these cases have been linked to the use of BP treatment in patients with cancer or osteoporosis $[2,3]$.

ONJ is an uncommon clinical entity defined as the presence of exposed bone that fails to heal after an appropriate intervention over a period of 6 or 8 weeks. ONJ was initially associated with the use of intravenous zoledronic acid, the most potent BP, but occurrences after the use of pamidronate and other oral BPs (alendronate, etidronate, ibandronate, risedronate, tiludronate) have also been reported. These reports were mostly case reports or case series without appropriate controls. More rigorous research design is clearly needed to confirm and estimate the size of the risk. Randomized clinical trial represents the highest level of evidence for causal relationship in clinical research. However, limited by the small sample size and short follow-up duration for a rare adverse event that requires long-term BP exposure, it is not easily identified. Restricted membership may further exclude some patients that are prone to develop such rare complications. Therefore, the risk of BP use on ONJ is better studied in a large population-based well-controlled observational study. Up to date, there are only a few such studies with inconsistent findings [4-17], warranting a systemic summary of current evidence and quantification the risk of ON or ONJ associated with the use of BPs.

\section{Methods}

\section{Search strategy}

We searched the following sources for the use of bisphosphonate and osteonecrosis: MEDLINE via PubMed was searched between 1975 and Aug 2012, and Embase was searched between 1992 and Aug 2012. We did not set restrictions on publication date, country, or language. Two authors conducted the database searches independently. Studies published in the MEDLINE database were identified by combining two separate queries composed of medical subject heading (MeSH) and text word (tw) keywords for exposure and outcome of interest. The exposure query was searched using the following exploded headings and independent terms: ["diphosphonates" (MeSH terms) OR "alendronate" (MeSH terms) OR bisphosphonate (tw) OR zoledronate (tw) OR pamidronate (tw) OR alendronate (tw) OR neridronate (tw) OR olpadronate (tw) OR ibandronate (tw) OR risedronate (tw) OR Aredia (tw) OR Zometa (tw) OR Boniva (tw) OR Actonel (tw) OR Aclasta (tw) OR Fosamax (tw)]. The outcome query was searched using exploded headings and independent terms for osteonecrosis: ["osteonecrosis" (MeSH terms) OR osteonecrosis (tw) OR Kienbock disease (tw) OR aseptic necrosis of bone (tw)]. We used manual restrictions by study type, the advanced automated methods in PubMed, to avoid unnecessary elimination of articles relevant to the search. We used a similar search strategy and search terms when searching in Embase. In addition, we searched reference lists of relevant articles manually for additional articles.

\section{Inclusion and exclusion criteria}

Two reviewers independently identified articles eligible for indepth examination by using the following inclusion and exclusion criteria. Observational studies were included if osteonecrosis was analyzed as an outcome of the study. The diagnosis of osteonecrosis was based on the physician diagnosis regarding medical records, clinical findings of exposed necrotic bone of jaw with compatible radiographic findings, or ICD-9 code or other diagnostic code for $\mathrm{ON}$ in a health claim database. Relevant exposures were defined as oral or intravenous use of bisphosphonates. We included studies on human subjects who were 15 years or older. The study types included were cohort and case-control studies. Case reports, case series, review articles, editorials, metaanalysis, clinical guidelines, and randomized controlled trials were excluded. We included studies on cancer patient populations; studies on osteoporosis or postsurgery patients were excluded. For those studies that reported the same cohort in multiple articles, we included only the most recent publication that met the inclusion criteria. Any discrepancies between reviewers on articles meriting inclusion were resolved by a consensus meeting of three authors. The study selection is summarized in Appendix 1 in Electronic supplementary material (ESM).

\section{Data extraction and synthesis}

We extracted data for study location, setting (e.g., population based, hospital based), population characteristics such as age range or underlying diseases, number of participants, type of BPs used in the study and route of administration, diagnosis of outcome, crude and adjusted effect sizes as available, and 
corresponding confidence intervals. We also recorded quality indicators of the study design, including the presence of appropriate controls and covariates adjusted for multivariate analysis. When studies were identified as containing pertinent data not included in the published article, we attempted to contact the authors to procure the missing data. When the authors did not respond and raw data were provided in the article, manual calculations of unadjusted effect estimates [odds ratio (OR)] were performed for inclusion in our metaanalysis. Otherwise, such analyses were excluded.

\section{Statistical analysis}

We conducted the meta-analysis of observational studies in our data extraction, analyses, and report according to the Preferred Reporting Items for Systematic Review and MetaAnalyses (PRISMA) guidelines [18]. We used the odds ratio as the measurement estimate for risk; when raw data or odds ratios were not available, we used the hazard ratio or risk ratio for analysis. Pooled ORs were computed as the MantelHaenszel-weighted average of the ORs for all included studies. Heterogeneity was measured by the Cochran Q statistic $(p<0.05)$ and quantified with the $I^{2}$ statistic [19]. The $I^{2}$ statistic describes the variation in the effect estimate that is attributable to heterogeneity across studies. The value of the $I^{2}$ statistic was used to select the appropriate pooling method as follows: fixed effects models were used for $I^{2}$ values less than $50 \%$ and for $I^{2}$ values greater than $50 \%$; random effects models were used [20]. Galbraith plots were used to visualize the impact of individual studies on the overall homogeneity test statistic [21]. Meta-regression was used to evaluate the amount of heterogeneity in the subgroup analysis. Publication bias was assessed by utilizing two methods. Begg and Egger tests were used to quantify the publication bias, and funnel plots were used to visualize the potential publication bias. Asymmetry of funnel plot indicated the reveal of publication bias [22]. To examine the potential effects of different methodological quality factors and adjustment for covariates, subgroup and sensitivity analyses were conducted. The metan, metabias, heterogi, and metareg macros were performed using Stata 11.0 (StataCorp, College Station, TX) as meta-analytic procedures. $p$ values of $<0.05$ were considered statistically significant.

\section{Results}

Search results and study characteristics

The flow of inclusion and exclusion are summarized in Appendix 1 in ESM. Using our search criteria, we identified 1,722 studies, of which 761 were from PubMed, and 961 from Embase. We excluded 1,377 citations based on screening titles and abstracts using predefined criteria mentioned in the method section. Five additional citations were identified from the reference lists. A total of 350 articles were retrieved from full-text review, and 342 were excluded due to various reasons, and these are detailed in Appendix 1 in ESM. Notably, the majority of studies were excluded due to a lack of appropriate controls. Finally, eight studies met the inclusion criteria. The eight included studies were comprised of 1,389 cases and 569,620 controls [4-17]. Of the included studies, two were case-control studies, and the remaining six were cohort studies. Details of the studies are summarized in Table 1. The studies varied with their settings, study populations, sample sizes, exposure and outcome definitions, and covariates adjusted in multivariate analysis. Breast cancer, lung cancer, prostate cancer, and multiple myeloma consisted of the largest part of study population. These are the four types of cancer with highest propensity for bone metastasis. The types of BPs and route of administration also varied with studies. Five studies reported effect estimate on oral BPs, and six reported effect estimates on intravenous (IV) BPs. Six of eight studies reported the predisposing factors for developing ONJ and ON. Dental extractions or trauma, poor oral hygiene, concurrent use of chemotherapeutic or antiangiogenic agents, or undergoing radiotherapy is the most commonly reported risk factors.

\section{Use of BPs and risk of ON}

When the results from all eight studies were combined, use of BPs was associated with an increased risk of ON (pooled OR 4.25; $95 \%$ confidence interval (CI) $3.67-5.36 ; I^{2}=0 \%$, Fig. 1a). Four studies reported only crude effect estimate without multivariate adjustment. Of the remaining four studies providing adjusted effect estimates, the pooled OR was slightly attenuated ( $4.22 ; 95 \%$ CI $3.21-5.54 ; I^{2}=0 \%$, Fig. 2).

\section{Use of BPs and risk of ONs in study design subgroups}

Five studies used the population-based claim databases or cancer registry, while three studies used medical records from the hospitals. Among the five population-based studies, the pooled OR is attenuated with an increased degree of homogeneity $\left(2.15 ; 95 \%\right.$ CI $\left.1.77-2.60 ; I^{2}=44.8 \%\right)$. The pooled OR is higher in the three studies using hospital records with a more rigid definition on the ONJ outcome (pooled OR $3.62 ; 95 \%$ CI $1.18-11.1 ; I^{2}=0 \%$ ) (Table 2).

Use of BPs and risk of ONs in different exposure subgroups

Use of oral BPs in cancer patients was associated with a small and nonsignificant risk for ONJ (pooled OR 1.18; $95 \% \mathrm{CI}$ $0.89-1.56 ; I^{2}=0 \%$ ). Use of IV BPs was associated with a 


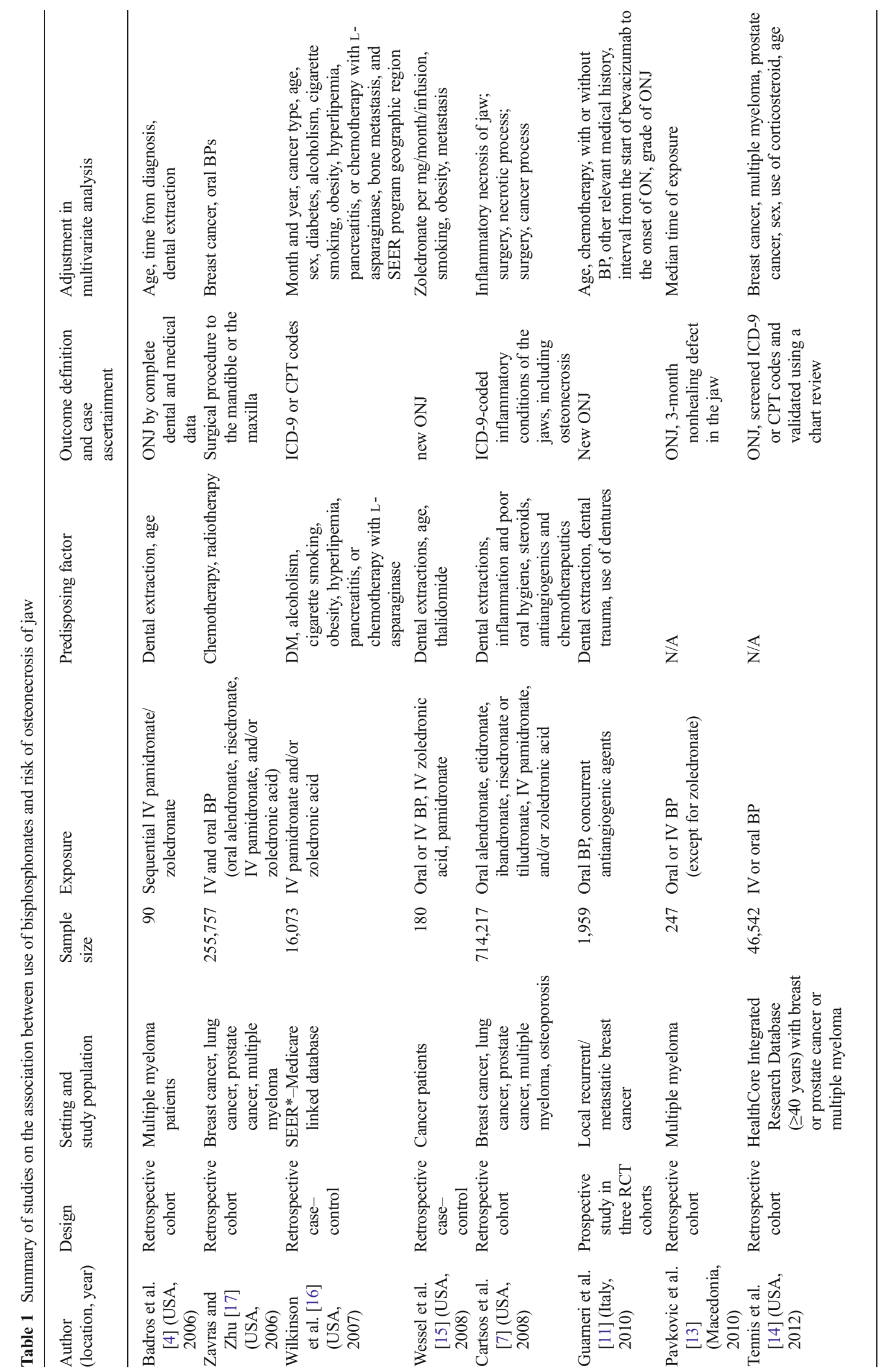


a

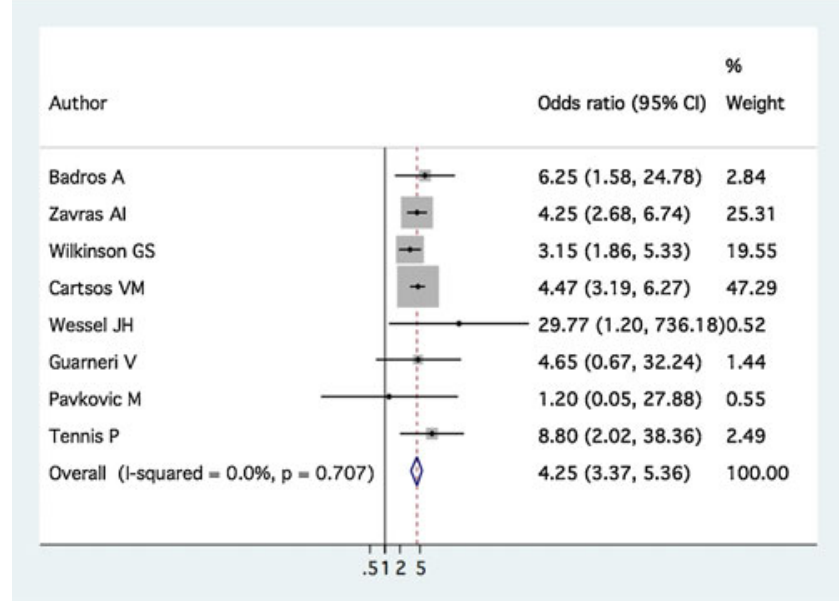

b

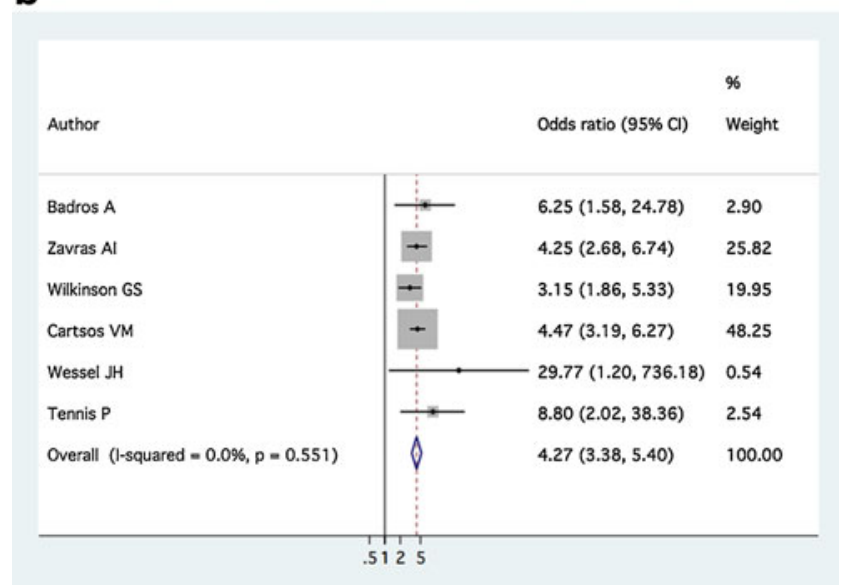

Fig. 1 Forest plot of the diagnostic odds ratios of studies using both oral and IV BPs (a) and IV BPs (b) for the detection an increased risk of ONJ

higher risk for ONJ (pooled OR 4.27; $95 \%$ CI 3.38-5.40; $I^{2}=0 \%$ Fig. 1b) (Table 2).

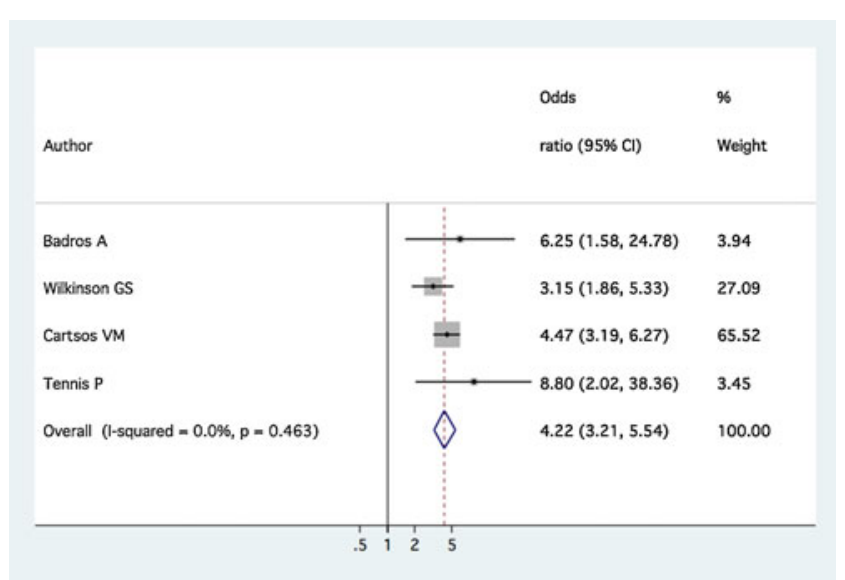

Fig. 2 Forest plot of the diagnostic odds ratios of studies using adjusted effect estimates for the detection an increased risk of ON among BP users
Table 2 Summary of subgroup analysis of studies of the association of $\mathrm{BPs}$ and risk of $\mathrm{ONJ}$

\begin{tabular}{|c|c|c|c|}
\hline Category & $\begin{array}{l}\text { Number of } \\
\text { studies }\end{array}$ & $\begin{array}{l}\text { Summary estimate } \\
(95 \% \mathrm{CI})\end{array}$ & $I^{2}(95 \% \mathrm{CI})$ \\
\hline All studies & 8 & $4.25(3.37-5.36)$ & $00.0(0.0-56.3)$ \\
\hline Adjusted studies & 4 & $4.22(3.21-5.54)$ & $00.0(0.0-67.9)$ \\
\hline $\begin{array}{l}\text { Population-based } \\
\text { studies }\end{array}$ & 5 & $2.15(1.77-2.60)$ & $44.8(0.0-78.3)$ \\
\hline $\begin{array}{l}\text { Hospital-based } \\
\text { studies }\end{array}$ & 3 & $3.62(1.18-11.13)$ & $00.0(0.0-72.9)$ \\
\hline IV BPs & 6 & $4.27(3.38-5.40)$ & $00.0(0.0-61.0)$ \\
\hline Oral BPs & 5 & $1.18(0.89-1.56)$ & $00.0(0.0-64.1)$ \\
\hline
\end{tabular}

Publication bias

There is no compelling evidence of publication bias in the overall analysis and subgroup analysis. Table 3 shows the results of statistical tests for publication bias.

Meta-regression analysis

A meta-regression analysis was performed to help explain the variation even after subgroup analysis. Table 4 shows the relative diagnostic odds ratio estimates from the metaregression analysis. Hospital-based studies and intravenous route of administration tend to increase the risk of ONJ, while adjusted studies were negatively associated with the risk estimate. None of the other covariates in the model reached a statistical significance.

\section{Discussion}

BPs are one of the most frequently prescribed drugs to treat cancer-related bone resorption. The use of BPs is consistently growing since its approval by the FDA. Unlike most medications, BPs are not totally excreted by the kidneys but are deposited within the bones. Accumulated literature has suggested a possible link between the use of BPs and a rare adverse effect, ONJ [19]. Due to the rarity of the ONJ, most published studies were isolated single case or retrospective case series reports without appropriate controls [19, 23]. The true impact of BPs on the incidence of ONJ at the population level has not been determined. Related literature has not been systemically reviewed and quantitatively summarized. In our

Table 3 Tests for publication bias and trim-and-fill ORs

\begin{tabular}{lll}
\hline Exposure & Begg $p$ value & Egger $p$ value \\
\hline Overall & 0.536 & 0.424 \\
Adjusted & 0.734 & 0.523 \\
\hline
\end{tabular}


Table 4 Exploration of heterogeneity in studies evaluating a risk of osteonecrosis among uses of bisphosphonates

\begin{tabular}{llll}
\hline Comparison & $\begin{array}{l}\text { Model } \\
\text { coefficient }\end{array}$ & $\begin{array}{l}\text { Relative } \\
\text { diagnostic } \\
\text { odds ratio }\end{array}$ & $p$ value \\
\hline $\begin{array}{c}\text { Adjusted studies vs. } \\
\text { unadjusted studies }\end{array}$ & $-0.05(0.29)$ & $0.95(0.43-2.10)$ & 0.86 \\
$\begin{array}{c}\text { Hospital-based studies vs. } \\
\text { population-based studies }\end{array}$ & $0.18(0.71)$ & $1.20(0.17-8.51)$ & 0.81 \\
$\begin{array}{c}\text { Intravenous vs. oral route of } \\
\text { administration }\end{array}$ & $0.34(0.95)$ & $1.40(0.10-19.60)$ & 0.74 \\
\hline
\end{tabular}

study of 0.5 million people in eight observational studies, we showed a statistically significant strong association between the use of BPs and the risk of ONJ in patients with cancer. The weighted summary OR for developing ON was 4.25 (3.375.36) for the use of BPs among cancer patients. Specifically, for cancer patients who received IV BPs, the odds ratio summary was 4.27 (95\% CI 3.38-5.40), and the incidence of ONJ may increase by $327 \%$ with IV BP prescription. The number needed to harm (NNH) was estimated as being 305 . The subgroup in which we could not find significant associations was in cancer patients receiving oral BPs.

The pathogenesis of ONJ remains to be elucidated [23-25]. Although animal studies have demonstrated some antiangiogenic properties of BPs, histological examination of most ONJ specimen did show adequate and patent blood vessels without evidence of ischemic necrosis of the bone [23, $24,26]$. Direct toxicity with induced apoptosis of osteocyte has also been suggested [23, 24, 26]. Histomorphometric studies showed defects in bone mineralization might also be a risk factor or BP-related ONJ [26, 27]. Recently, a specific polymorphism of RBMS3, a protein involved in the regulation of collagen type I in fibroblasts, has been suggested to associate with BP-related ONJ in a genome-wide association study [28]. In addition, infection was commonly observed in cases of ONJ and has been suggested to play a synergistic or triggering role for the pathogenesis of ONJ [29-31].

Several preexisting risk factors influencing the normal bone metabolism have been reported in the included studies. Increasing age, infection, dental extractions or trauma, or concurrent use of chemotherapeutic or antiangiogenic agents are the most common risk factors. Of note, dental trauma such as dental extraction, dentures, or dental trauma during intubation procedure has been clearly shown as the strongest precipitating factor for BP-related ONJ. Before starting BP therapy, a comprehensive dental examination should be arranged. The goal of the dental care would be to eliminate of all potential sites of infection and to attain a state of good oral and dental health during the course of IV BP therapy. For patients who already received bisphosphonate therapy, avoiding dental extraction is important since the most common history of BP-related ONJ is delayed or absent of healing after dental extraction. For those dental procedures that are unavoidable, some experts recommend withdrawal of BP therapy for at least 3 months before dental surgery, although the optimal duration for BP withdrawal remains unknown. Discontinuation of BP therapy may not affect the BP already incorporated into the bone but may avoid the antiangiogenic effect of BP which is harmful for the healing of traumatized oral tissue. In the early stages of ONJ, radiographic manifestations can be vague. Severe jaw pain is usually the earliest symptoms, when necrotic bone has become infected. Therefore, early dental consultation is necessary for patients with dental symptoms during IV BP therapy. For those who consider starting the use of BP either for therapeutic or preventive purpose, the aforementioned conditions should be considered as relative contraindications, and good oral hygiene along with regular dental care is the best way to lower risk.

Five population-based studies used medical claims database and were associated with an attenuated risk estimate with increased heterogeneity. Some characteristics of medical claims database study may help explain the phenomenon. First, there is no ICD-9 code for ONJ. Investigators have to use operations on the jaw or facial bones or inflammatory conditions of the jaw as surrogate outcomes, and misclassification was likely. In the case of random misclassification, the risk estimates tend to be biased toward the null. In addition, different outcome definitions used may have increased the heterogeneity of the risk estimates. Secondly, patients with ONJ may have been treated by either dentists or oral surgeons. Unless both treatments were covered in a medical insurance plan, either a dental plan claims database or a medical plan claims database would not capture the full spectrum of the ONJ events. This could lead to the underestimation of the number of ONJ cases among both BP users and nonusers.

Despite the physiological plausibility and statistically significant and homogeneous results in this large sample size meta-analysis, several limitations to this study should be considered before a firm conclusion can be drawn. Firstly, the induction period of ONJ after BP exposure is unknown. However, the diagnosis of $\mathrm{ONJ}$, by definition, requires a minimum of 6 weeks of exposed bone. Most of the included studies did not take this induction period into consideration for exposure classification, and there is a possibility of exposure misclassification. Random misclassification may attenuate the risk, and nonrandom misclassification may have biased the results in an unpredictable direction. Secondly, the modality and criteria of diagnosis of ONJ varied across included studies. Hospital-based studies used clinical records for outcome ascertainment; therefore, the diagnosis of ONJ may be more reliable. Population-based studies were based on health claim data that used a set of ICD-9 codes to capture ONJ events. We found the risk estimate for population-based studies (pooled OR 2.15; $95 \%$ CI 1.77-2.60) which was 
lower than that (pooled OR 3.62; $95 \%$ CI 1.18-11.1) of hospital-based studies. Therefore, there is a tendency that ICD-9 codes may underestimate the incidence of ONJ. Lastly, the duration and dosage of BP use are important determinant factors for ONJ but have not been investigated in most of these studies. The reported median/mean duration of BP exposure in the included studies ranged from 10 months to 6 years. Unless patient-level data are available, analysis of doseresponse relationship, defining a specific BPs with higher risk or defining safety range for BP use, is not possible.

In conclusion, our meta-analysis of more than 0.5 million patients indicated that the use of IV BPs among cancer patients is associated with four times risk of developing ONJ. Given the low baseline incidence, the absolute risk for those who received IV BP in cancer patients is low. For the majority of people considering the use of BPs for prevention of cancer-related fractures, these results should reassure them of the importance of weighing the benefits of BPs against this rare but severe complication. For those whose use of BPs is unavoidable, our results emphasize the importance of assessing oral health before initiating therapy and avoid dental procedures during the active phase of intravenous BP therapy.

Acknowledgments We thank the Medical Wisdom Corporation for the assistance with statistical analysis. We also thank Yin Ling Tan, a medical student from the National Taiwan University Hospital, for her help at the early stage of inclusion and exclusion of eligible studies. This work was supported by a grant from the National Science Council (NSC) (1002341-B-002-138-MY3NSC).

Conflict of interest None declared.

\section{References}

1. Zavras AI (2011) The impact of bisphosphonates on oral health: lessons from the past and opportunities for the future. Ann N Y Acad Sci 1218:55-61

2. Marx RE (2003) Pamidronate (Aredia) and zoledronate (Zometa) induced avascular necrosis of the jaws: a growing epidemic. J Oral Maxillofac Surg 61:1115-1117

3. Ruggiero SL, Mehrotra B, Rosenberg TJ, Engroff SL (2004) Osteonecrosis of the jaws associated with the use of bisphosphonates: a review of 63 cases. J Oral Maxillofac Surg 62:527-534

4. Badros A, Weikel D, Salama A, Goloubeva O, Schneider A, Rapoport A, Fenton R, Gahres N, Sausville E, Ord R, Meiller T (2006) Osteonecrosis of the jaw in multiple myeloma patients: clinical features and risk factors. J Clin Oncol 24:945-952

5. Baillargeon J, Kuo YF, Lin YL, Wilkinson GS, Goodwin JS (2011) Osteonecrosis of the jaw in older osteoporosis patients treated with intravenous bisphosphonates. Ann Pharmacother 45:1199-1206

6. Barasch A, Cunha-Cruz J, Curro FA, Hujoel P, Sung AH, Vena D, Voinea-Griffin AE, Beadnell S, Craig RG, DeRouen T, Desaranayake A, Gilbert A, Gilbert GH, Goldberg K, Hauley R, Hashimoto M, Holmes J, Latzke B, Leroux B, Lindblad A, Richman J, Safford M, Ship J, Thompson VP, Williams OD, Yin W (2011) Risk factors for osteonecrosis of the jaws: a case-control study from the CONDOR dental PBRN. J Dent Res 90:439-444
7. Cartsos VM, Zhu S, Zavras AI (2008) Bisphosphonate use and the risk of adverse jaw outcomes: a medical claims study of 714,217 people. J Am Dent Assoc 139:23-30

8. Cooper C, Steinbuch M, Stevenson R, Miday R, Watts NB (2010) The epidemiology of osteonecrosis: findings from the GPRD and THIN databases in the UK. Osteoporos Int 21:569-577

9. Etminan M, Aminzadeh K, Matthew IR, Brophy JM (2008) Use of oral bisphosphonates and the risk of aseptic osteonecrosis: a nested case-control study. J Rheumatol 35:691-695

10. Fellows JL, Rindal DB, Barasch A, Gullion CM, Rush W, Pihlstrom DJ, Richman J (2011) ONJ in two dental practice-based research network regions. J Dent Res 90:433-438

11. Guarneri V, Miles D, Robert N, Dieras V, Glaspy J, Smith I, Thomssen C, Biganzoli L, Taran T, Conte P (2010) Bevacizumab and osteonecrosis of the jaw: incidence and association with bisphosphonate therapy in three large prospective trials in advanced breast cancer. Breast Canc Res Treat 122:181-188

12. Kallmes DF, Rad AE, Gray LA, McDonald RJ, Clarke BL (2009) Bisphosphonates are not associated with vertebral osteonecrosis. J Bone Miner Metabol 27:452-455

13. Pavkovic M, Petrushevska G, Jovanovic R, Karanfilski O, Cevreska L, Stankovic S, Stojanovic A (2010) Osteonecrosis of the jaw in patients with multiple myeloma treated with bisphosphanates. Prilozi 31:39-49

14. Tennis P, Rothman KJ, Bohn RL, Tan H, Zavras A, Laskarides C, Calingaert B, Anthony MS (2012) Incidence of osteonecrosis of the jaw among users of bisphosphonates with selected cancers or osteoporosis. Pharmacoepidemiol Drug Saf 21:810-817

15. Wessel JH, Dodson TB, Zavras AI (2008) Zoledronate, smoking, and obesity are strong risk factors for osteonecrosis of the jaw: a casecontrol study. J Oral Maxillofac Surg 66:625-631

16. Wilkinson GS, Kuo YF, Freeman JL, Goodwin JS (2007) Intravenous bisphosphonate therapy and inflammatory conditions or surgery of the jaw: a population-based analysis. J Natl Canc Inst 99:1016-1024

17. Zavras AI, Zhu S (2006) Bisphosphonates are associated with increased risk for jaw surgery in medical claims data: is it osteonecrosis? J Oral Maxillofac Surg 64:917-923

18. Moher D, Liberati A, Tetzlaff J, Altman DG (2009) Preferred reporting items for systematic reviews and meta-analyses: the PRISMA statement. BMJ 339:b2535

19. Lijmer JG, Bossuyt PM, Heisterkamp SH (2002) Exploring sources of heterogeneity in systematic reviews of diagnostic tests. Stat Med 21:1525-1537

20. DerSimonian R, Laird N (1986) Meta-analysis in clinical trials. Control. Clin Trials 7:177-188

21. Galbraith RF (1988) A note on graphical presentation of estimated odds ratios from several clinical trials. Stat Med 7:889-894

22. Song F, Khan KS, Dinnes J, Sutton AJ (2002) Asymmetric funnel plots and publication bias in meta-analyses of diagnostic accuracy. Int J Epidemiol 31:88-95

23. Filleul O, Crompot E, Saussez S (2010) Bisphosphonate-induced osteonecrosis of the jaw: a review of 2,400 patient cases. J Cancer Res Clin Oncol 136:1117-1124

24. Allen MR, Burr DB (2009) The pathogenesis of bisphosphonaterelated osteonecrosis of the jaw: so many hypotheses, so few data. J Oral Maxillofac Surg 67:61-70

25. Reid IR, Cornish J (2012) Epidemiology and pathogenesis of osteonecrosis of the jaw. Nat Rev Rheumatol 8:90-96

26. Favia G, Pilolli GP, Maiorano E (2009) Histologic and histomorphometric features of bisphosphonate-related osteonecrosis of the jaws: an analysis of 31 cases with confocal laser scanning microscopy. Bone 45:406-413

27. Hoefert S, Schmitz I, Tannapfel A, Eufinger H (2010) Importance of microcracks in etiology of bisphosphonate-related osteonecrosis of the jaw: a possible pathogenetic model of symptomatic and nonsymptomatic osteonecrosis of the jaw based on scanning electron microscopy findings. Clin Oral Investig 14:271-284 
28. Nicoletti P, Cartsos VM, Palaska PK, Shen Y, Floratos A, Zavras AI (2012) Genomewide pharmacogenetics of bisphosphonate-induced osteonecrosis of the jaw: the role of RBMS3. Oncologist 17:279-287

29. Kumar SK, Gorur A, Schaudinn C, Shuler CF, Costerton JW, Sedghizadeh PP (2010) The role of microbial biofilms in osteonecrosis of the jaw associated with bisphosphonate therapy. Curr Osteoporos Rep 8:40-48
30. Marx RE, Tursun R (2012) Suppurative osteomyelitis, bisphosphonate induced osteonecrosis, osteoradionecrosis: a blinded histopathologic comparison and its implications for the mechanism of each disease. Int J Oral Maxillofac Surg 41:283-289

31. Sedghizadeh PP, Kumar SK, Gorur A, Schaudinn C, Shuler CF, Costerton JW (2009) Microbial biofilms in osteomyelitis of the jaw and osteonecrosis of the jaw secondary to bisphosphonate therapy. $\mathrm{J}$ Am Dent Assoc 140:1259-1265 